\title{
Tasa de infección en 212 pacientes consecutivos tratados mediante descompresión tubular mininvasiva de la columna lumbar
}

\author{
Nicolás Coombes, Eduardo Galaretto, Juan Pablo Guyot, Norberto Fernández, Cristian Fuster \\ Axial Grupo Médico, Ciudad Autónoma de Buenos Aires, Argentina
}

\begin{abstract}
RESUMEN
Introducción: Las infecciones del sitio quirúrgico pueden requerir tratamientos prolongados con antibióticos, una estancia hospitalaria más prolongada y operaciones repetidas. Las incisiones pequeñas y la posibilidad de evitar la disección subperióstica en la cirugía mininvasiva pueden reducir, al mínimo, el riesgo de infecciones posoperatorias. Sin embargo, los estudios publicados sobre infecciones después de procedimientos mininvasivos son escasos. El objetivo de este estudio fue evaluar retrospectivamente una serie de pacientes operados, de manera consecutiva, con una técnica mininvasiva y la incidencia de infección posoperatoria. Materiales y Métodos: Se incluyó a los pacientes sometidos a cirugía lumbar posterior con retractores tubulares y microscopio, en nuestro Servicio, entre enero de 2015 y enero de 2018. Los procedimientos realizados fueron discectomías tubulares, descompresiones para la estenosis de canal y resecciones de quistes sinoviales. La incidencia de infección del sitio quirúrgico se calculó y comparó con el rango de tasas de infección del sitio quirúrgico publicado. Resultados: Se realizaron cirugías no instrumentadas (discectomías, descompresiones) en 212 pacientes. La media de la edad era de 62.4 años y la relación hombre:mujer, de 1,27:1. Solo un paciente sufrió una infección del sitio quirúrgico, diagnosticada el día 9 poscirugía, que fue tratada mediante lavado y limpieza de la herida. Conclusión: La tasa de infección fue del 0,47\%. La cirugía mininvasiva puede disminuir notablemente la tasa de infección del sitio quirúrgico y puede ser una herramienta eficaz para reducir, al mínimo, los costos hospitalarios.

Palabras clave: Procedimientos quirúrgicos mininvasivos; descompresión; discectomía; infecciones de heridas quirúrgicas.

Nivel de Evidencia: IV
\end{abstract}

Infection rate in $\mathbf{2 1 2}$ consecutive patients treated by minimally invasive lumbar tubular decompression

\begin{abstract}
Introduction: Surgical site infections (SSI) can lead to prolonged antibiotic treatments, increased hospital stays, and repeated operations. Small incisions and the possibility of avoiding subperiosteal dissection in minimally invasive surgery can minimize the risk of postoperative infections. However, there is a shortage of literature on infections after mini-invasive procedures. Objective: To evaluate retrospectively a series of patients consecutively operated on with minimally invasive technique and the incidence of postoperative infection. Materials and Methods: All patients undergoing posterior lumbar surgery with tubular retractors and microscope in our department from January 2015 to January 2018 were included. The procedures performed included tubular discectomies, lumbar stenosis decompressions, and synovial cyst resections. The incidence of postoperative SSIs was calculated and compared with the literature range for SSI rates. Results: A total of 212 patients underwent non-instrumented surgeries (discectomies, decompressions). The mean age was 62.4 years with a male:female ratio of 1.27:1. Only one patient had SSI, which was diagnosed on day 9 and treated by reoperation, surgical toilet of the wound, and antibiotic therapy. Conclusion: The infection rate was $0.47 \%$. Minimally invasive surgery can significantly reduce the SSI rate and can be an effective tool in minimizing hospital costs.
\end{abstract}

Key words: Minimally invasive surgical procedures, decompression, discectomy, surgical wound infections.

Level of Evidence: IV 


\section{INTRODUCCIÓN}

Las infecciones del sitio quirúrgico (ISQ) son complicaciones muy temidas por los cirujanos de columna, ya que las tasas de morbimortalidad son significativas, oscilan entre el 1,9\% y el 5,5\%. ${ }^{1-4}$ Esta variabilidad podría estar relacionada con un subregistro de las complicaciones infecciosas que no se informan y generan resultados muy variables. ${ }^{5-7}$

Las ISQ espinales pueden ser complicaciones graves, ya que pueden ser difíciles de manejar y requerir hospitalización prolongada, antibioticoterapia específica por vía endovenosa, cirugías repetidas para el desbridamiento de la herida o el retiro del implante. ${ }^{8}$

Es muy aconsejable reducir, al mínimo, las ISQ. Las técnicas quirúrgicas mininvasivas de la columna vertebral reducen el abordaje necesario para acceder a la columna vertebral y disminuyen la destrucción del tejido, la pérdida de sangre, la estancia hospitalaria y la morbilidad posoperatoria. ${ }^{9}{ }^{10}$ Solo unos pocos estudios han evaluado la incidencia de ISQ después de una cirugía mininvasiva de la columna vertebral. ${ }^{11-13}$

El objetivo de este estudio fue evaluar la tasa de infección en una serie de pacientes tratados, de forma consecutiva, por un mismo equipo quirúrgico, con descompresiones tubulares lumbares mininvasivas sin instrumentación; analizar los resultados y compararlos con las estadísticas publicadas.

\section{MATERIALES Y MÉTODOS}

Se analizó, en forma retrospectiva, a todos los pacientes consecutivos que se sometieron a una cirugía mininvasiva de la columna lumbar, entre enero de 2015 y diciembre de 2018. Se definió como cirugía mininvasiva de la columna lumbar a aquellas realizadas con sistemas de retractores tubulares asistidas por microscopia. Todos los pacientes fueron operados por el mismo equipo quirúrgico.

El protocolo prequirúrgico incluyó la descontaminación domiciliaria con baños de clorhexidina jabonosa, durante tres días, antes de la internación.

El paciente fue colocado en posición prona en la mesa de operaciones y recibió anestesia general. Se lavó la zona quirúrgica con paños de clorhexidina y se la rasuró durante la cirugía, si era necesario. Se colocaron campos operatorios de material descartable autoadhesivos, de sentido caudal a cefálico; en el último paso, se cubrió la zona quirúrgica con un campo autoadhesivo yodado. Hasta el último paso, el equipo quirúrgico utilizó doble guante. Todos los procedimientos se realizaron utilizando retractores tubulares de 18 a 26 mm de diámetro (Sistema METRx, Medtronics, Memphis, TN, EE.UU.). Como regla, se administró el antibiótico preoperatorio (cefalosporina de tercera generación) una hora antes del procedimiento. ${ }^{8}$ El microscopio y el intensificador de imágenes se cubrieron adecuadamente con fundas estériles. Las discectomías tubulares se llevaron a cabo con tubos de entre 18 y $22 \mathrm{~mm}$ de diámetro, y las descompresiones tubulares, en los canales estrechos de 22 y $26 \mathrm{~mm}$ de diámetro. El antibiótico se repitió solo una vez después de la cirugía. Los pacientes se movilizaron de 3 a 4 h poscirugía. Los pacientes con lesiones durales y fugas de líquido cefalorraquídeo se movilizaron el primer día poscirugía.

La infección posoperatoria se definió según las características clínicas, que incluían: salida persistente de líquido por la herida, dolor lumbar y parámetros inflamatorios elevados (leucocitosis, eritrosedimentación, proteína $\mathrm{C}$ reactiva) y la evaluación por resonancia magnética. Se consideró que los pacientes con infecciones se habían curado solo cuando dos marcadores inflamatorios consecutivos eran negativos, durante un período intermedio de, al menos, 15 días y acompañado de mejoría clínica.

\section{RESULTADOS}

Se incluyó a 212 pacientes (119 hombres y 93 mujeres) para un total de 257 niveles descomprimidos. La media de la edad antes de la cirugía era de 62.4 años (rango 14-86) y la relación hombre:mujer, de 1,27:1.

Los diagnósticos eran canal estrecho (103 casos, 48,58\%), hernia de disco lumbar (74 casos, 34,9\%) y quistes sinoviales (35 casos, 16,5\%).

El tiempo operatorio promedio fue de $60 \pm 25.4 \mathrm{~min}$ (para cada nivel). En el 91,6\% de los pacientes, se realizó un abordaje único y, en el 8,4\%, un abordaje múltiple (más de 2). El tiempo transcurrido entre el inicio de la cirugía y la detección de la infección fue de 9 días.

Se diagnosticó una sola infección, que representó el 0,47\% $(\mathrm{n}=1)$. Se trató de un caso de revisión, ya que el paciente había sido sometido a una cirugía primaria un año antes. En ese procedimiento, se planteó un abordaje combinado, porque los segmentos por descomprimir eran más de uno, en un paciente con estenosis multinivel.

Una vez diagnosticada la infección, se procedió al lavado y al desbridamiento quirúrgico durante el cual se tomaron muestras para cultivo bacteriológico. El paciente permaneció internado y recibió antibióticos. En el cultivo, se aisló Staphylococcus aureus sensible a la meticilina y se le indicó tratamiento antibiótico ambulatorio. 


\section{DISCUSIÓN}

Las ISQ en la cirugía de columna vertebral pueden desencadenar complicaciones catastróficas y empeorar los resultados clínicos. ${ }^{1,2}$ Las tasas informadas de ISQ después de la cirugía abierta de columna varían del $0,7 \%$ al 5,5\% $\%^{1-6}$ y las tasas informadas para descompresiones tubulares sin instrumentación oscilan entre el 0,09\% y el 0,22\%. ${ }^{11-13}$

Las cirugías abiertas de columna demandan incisiones grandes, la disección extensa de tejidos blandos y una retracción más amplia. La morbilidad iatrogénica resultante está muy bien establecida. ${ }^{14}$ Se ha producido un cambio de paradigma desde la resección subperióstica y la retracción muscular excesiva hacia la dilatación progresiva de tejidos blandos utilizando los dilatadores secuenciales y los retractores tubulares. ${ }^{7}, 15$ Desde que Foley y Smith comenzaron a usar los retractores tubulares, a finales de la década de 1990, varios autores han tratado de determinar las tasas de infección después de la cirugía mininvasiva. ${ }^{11-13,16}$

Las razones probables de las tasas más bajas de infección tras una cirugía mininvasiva son el pequeño abordaje quirúrgico, la reducción del área de superficie expuesta y la disminución del espacio muerto. ${ }^{15}$ Menos retracción muscular significa menos isquemia, menos necrosis y mejor potencial de curación de las heridas. Un campo quirúrgico más restringido conduce a una menor exposición a posibles patógenos y disminuye los trastornos y la flora cutáneos, ya que están protegidos por un tubo. ${ }^{11}$ La estancia hospitalaria más corta observada con la cirugía mininvasiva también puede reducir la exposición a microorganismos patógenos adquiridos en el lugar de internación. Sin embargo, preocupa la curva de aprendizaje que enfrenta un cirujano durante la experiencia inicial con estos sistemas. ${ }^{12,17}$ La mayor duración operativa y la curva de aprendizaje empinada pueden modificar las tasas de infección observadas en diferentes centros. Por lo tanto, solo se puede emitir una declaración de consenso después de que se establezca evidencia concluyente sobre una reducción de las tasas de ISQ en los centros y cirujanos de columna en todo el mundo.

El único episodio en esta serie fue diagnosticado en el posoperatorio temprano (9 días) y el microorganismo fue $S$. aureus, esto coincide con lo publicado en otras series. ${ }^{9}, 13,14$

Se estima que los pacientes sometidos a cirugía espinal abierta tienen 5,77 veces más probabilidades de desarrollar ISQ que aquellos operados mediante los abordajes mininvasivos. ${ }^{14}$

O'Toole y cols. evaluaron las ISQ después de una cirugía mininvasiva de columna e informaron una incidencia del $0,10 \%$ en las cirugías no instrumentadas. ${ }^{13}$ Shousha y cols., en un gran estudio de 4350 casos de cirugías no instrumentadas por abordaje mininvasivo, comunicaron una tasa de infección del $0,09 \%{ }^{11}$

De acuerdo con la bibliografía, nuestras observaciones refuerzan la conclusión de que la cirugía mininvasiva de columna reduce la tasa de ISQ y también la morbilidad posoperatoria. ${ }^{4,8,9}$

Aunque el número de pacientes incluidos en nuestra serie fue mucho menor comparado con el de las series publicadas, la tasa de infección coincide con lo comunicado en la bibliografía, ya que si se toman como referencia las cirugías primarias, la tasa de infección fue del 0\%, pues esta complicación apareció en una etapa de revisión.

El intensificador de imágenes juega un papel importante en la ejecución exitosa de la cirugía. En nuestra práctica, cubrimos solo la parte superior del intensificador y mantenemos su esterilidad durante todo el procedimiento quirúrgico. La porción inferior del intensificador es la que sufre un cambio constante de posición que puede ser una fuente potencial de contaminación. Recién cubrimos la parte inferior del brazo $\mathrm{C}$ cuando el intensificador se coloca para una exposición lateral.

Bible y cols. estudiaron la esterilidad de los microscopios operativos y observaron que estos aparatos son una fuente importante de contaminación. Colocar el microscopio con fundas estériles y el uso de precauciones asépticas mientras se maneja el ocular ayudan a reducir la contaminación. ${ }^{18}$ Nos cambiamos los guantes cada vez que manipulamos el ocular óptico y evitamos el contacto con la parte de la funda por encima del ocular óptico.

Las infecciones después de la cirugía abierta de columna aumentan cuatro veces el costo de la atención médica. ${ }^{14} \mathrm{Al}$-Khouja y cols. reportaron una reducción del $33 \%$ al 2,54\% en el costo entre la cirugía mininvasiva y la cirugía abierta de columna. ${ }^{17}$ Por lo tanto, en la era actual de la rentabilidad y el análisis de costos, la cirugía mininvasiva de columna tiene un lugar prometedor para reducir el costo general de la atención médica.

Este estudio tuvo algunas limitaciones. Solo un paciente sufrió una infección (ya había sido operado); por lo tanto, no se puede extraer una conclusión válida con respecto a los factores de riesgo. La muestra es sustancialmente más pequeña que las publicadas, no contamos con un grupo de control sometido a cirugía abierta de columna, ya que todos los pacientes fueron operados utilizando técnicas quirúrgicas mininvasivas. Consideramos que se requieren grandes ensayos de casos y controles para una evaluación adicional. 


\section{CONCLUSIONES}

La tasa de infección después de la cirugía espinal asistida por microscopia tubular fue muy baja $(0,47 \%)$. La técnica mininvasiva reduce notablemente el riesgo de infección posoperatoria al compararla con otras series grandes publicadas sobre cirugía abierta de columna, puede ser una herramienta eficaz para reducir, al mínimo, los costos hospitalarios y la morbimortalidad del paciente.

Conflicto de intereses: Los autores no declaran conflictos de intereses.

ORCID de E. Galaretto: https://orcid.org/0000-0003-4121-8228 ORCID de J. P. Guyot: https://orcid.org/0000-0001-6524-4426
ORCID de N. Fernández: https://orcid.org/0000-0002-5250-5399 ORCID de C. Fuster: https://orcid.org/0000-0002-5293-0846

\section{BIBLIOGRAFÍA}

1. Beiner JM, Grauer J, Kwon BK, Vaccaro AR. Postoperative wound infections of the spine. Neurosurg Focus 2003; 15:E14. https://doi.org/10.3171/foc.2003.15.3.14

2. Olsen MA, Mayfield J, Lauryssen C, Polish LB, Jones M, Vest J, et al. Risk factors for surgical site infection in spinal surgery. J Neurosurg 2003;98(2 Suppl):149-55. https://doi.org/10.3171/spi.2003.98.2.0149

3. Collins I, Wilson-MacDonald J, Chami G, Burgoyne W, Vineyakam P, Berendt T, et al. The diagnosis and management of infection following instrumented spinal fusion. Eur Spine J 2008;17(3):445-50. https://doi.org/10.1007/s00586-007-0559-8

4. Valentini LG, Casali C, Chatenoud L, Chiaffarino F, Uberti-Foppa C, Broggi G. Surgical site infections after elective neurosurgery: a survey of 1747 patients. Neurosurgery 2008;62(1):88-95. https://doi.org/10.1227/01.NEU.0000311065.95496.C5

5. Olsen MA, Nepple JJ, Riew KD, Lenke LG, Bridwell KH, Mayfield J, et al. Risk factors for surgical site infection following orthopaedic spinal operations. J Bone Joint Surg Am 2008;90(1):62-69. https://doi.org/10.2106/JBJS.F.01515

6. Pappou IP, Papadopoulos EC, Sama AA, Girardi FP, Cammisa FP. Postoperative infections in interbody fusion for degenerative spinal disease. Clin Orthop Relat Res 2006;444:120-8. https://doi.org/10.1097/01.blo.0000203446.06028.b5

7. Friedman ND, Sexton DJ, Connelly SM, Kaye KS. Risk factors for surgical site infection complicating laminectomy. Infect Control Hosp Epidemiol 2007;28:1060-5. https://doi.org/10.1086/519864

8. Weinstein MA, McCabe JP, Cammisa FP Jr. Postoperative spinal wound infection: a review of 2,391 consecutive index procedures. J Spinal Disord 2000;13(5):422-6. https://doi.org/10.1097/00002517-200010000-00009

9. Fang A, Hu SS, Endres N, Bradford DS. Risk factors for infection after spinal surgery. Spine (Phila Pa 1976) 2005;30(12):1460-5. https://doi.org/10.1097/01.brs.0000166532.58227.4f

10. Kanayama M, Hashimoto T, Shigenobu K, Oha F, Togawa D. Effective prevention of surgical site infection using a Centers for Disease Control and Prevention guideline-based antimicrobial prophylaxis in lumbar spine surgery. J Neurosurg Spine 2007;6:327-9. https://doi.org/10.3171/spi.2007.6.4.7

11. Shousha M, Cirovic D, Boehm H. Infection rate after minimally invasive noninstrumented spinal surgery based on 4350 procedures. Spine (Phila Pa 1976) 2015;40(3):201-5. https://doi.org/10.1097/BRS.0000000000000690

12. Brodano GB, Martikos K, Lolli F, Gasbarrini A, Cioni A, Bandiera S, et al. Transforaminal lumbar interbody fusion in degenerative disk disease and spondylolisthesis grade I: minimally invasive versus open surgery. J Spinal Disord Tech 2015;28(10):E559-64. https://doi.org/10.1097/BSD.0000000000000034

13. O'Toole JE, Eichholz KM, Fessler RG. Surgical site infection rates after minimally invasive spinal surgery. J Neurosurg Spine 2009;11(4):471-6. https://doi.org/10.3171/2009.5.SPINE08633

14. Calderone RR, Garland DE, Capen DA, Oster H. Cost of medical care for postoperative spinal infections. Orthop Clin North Am 1996;27(1):171-82. https://pubmed.ncbi.nlm.nih.gov/8539047/ 
15. Fessler RG, O’Toole JE, Eichholz KM, Perez-Cruet MJ. The development of minimally invasive spine surgery. Neurosurg Clin N Am 2006;17(4):401-9. https://doi.org/10.1016/j.nec.2006.06.007

16. Ee WW, Lau WL, Yeo W, Von Bing Y, Yue WM. Does minimally invasive surgery have a lower risk of surgical site infections compared with open spinal surgery? Clin Orthop Relat Res 2014;472(6):1718-24. https://doi.org/10.1007/s11999-013-3158-5

17. Al-Khouja LT, Baron EM, Johnson JP, Kim TT, Drazin D. Cost-effectiveness analysis in minimally invasive spine surgery. Neurosurg Focus 2014;36(6):E4. https://doi.org/10.3171/2014.4.FOCUS1449

18. Bible JE, O’Neill KR, Crosby CG, Schoenecker JG, McGirt MJ, Devin CJ. Microscope sterility during spine surgery. Spine (Phila Pa 1976) 2012;37(/):623-7. https://doi.org/10.1097/BRS.0b013e3182286129 